\title{
Factores determinantes de la satisfacción del paciente con el tratamiento para la disfunción eréctil
}

\author{
Delgado Martín JA*, Blázquez Izquierdo J*, Silmi Moyano A*, Martínez E**. \\ *Servicio de Urología. Hospital Clínico San Carlos. Madrid. **Pfizer S.A.
}

Actas Urol Esp. 2008;32(10):995-1003

\begin{abstract}
RESUMEN
FACTORES DETERMINANTES DE LA SATISFACCIÓN DEL PACIENTE CON EL TRATAMIENTO PARA LA DISFUNCIÓN ERECTIL Introducción: La disfunción eréctil (DE) se asocia a estados depresivos, de ansiedad y pérdida de autoestima, empeorando la calidad de vida del paciente. El presente estudio tuvo por objetivo, identificar y evaluar los aspectos más relevantes para el paciente en relación con la satisfacción con el tratamiento para la DE, analizar cuales de éstos tienen mayor impacto sobre la autoestima y la satisfacción con diferentes aspectos de la vida

Métodos: Estudio epidemiológico, transversal, multicéntrico y observacional. Se incluyeron hombres españoles mayores de 18 años en tratamiento para la DE con cualquier fármaco oral, al menos 4 semanas antes del comienzo del estudio. El coeficiente de correlación de Pearson fue calculado entre los aspectos importantes para el paciente al valorar su satisfacción con el tratamiento (preguntas 4 a 11 del "Erectile Dysfunction inventory of treatment satisfaction", EDITS) y la satisfacción con el tratamiento (pregunta 1 EDITS), la subescala de autoestima del cuestionario "The Self-esteem and Relationship" (SEAR) y el cuestionario "Fugl-Meyer life satisfaction Check List" (LISAT-8).

Resultados: Se incluyeron un total de 1.646 pacientes. Los aspectos de mayor relevancia en relación con la satisfacción con el tratamiento para la DE, fueron: duración de los efectos ( $\mathrm{r}=0,7311 \mathrm{p} \leq 0,0001)$, rapidez de acción del fármaco ( $\mathrm{r}=0,7088 \mathrm{p} \leq 0,0001)$, confianza en poder mantener relaciones sexuales $(\mathrm{r}=0,7021 \mathrm{p} \leq 0,0001)$ y satisfacción de la pareja ( $\mathrm{r}=0,692 \mathrm{p} \leq 0,0001)$. Los aspectos que mayor impacto tuvieron sobre la autoestima fueron: duración de los efectos ( $\mathrm{r}=0,6254 \mathrm{p} \leq 0,0001)$ y confianza en poder mantener relaciones sexuales $(\mathrm{r}=0,6372 \mathrm{p} \leq 0,0001)$. Los aspectos que mayor impacto tuvieron sobre la mejora de diferentes aspectos de la vida (LISAT-8) fueron: facilidad de uso ( $\mathrm{r}=0,4372 \mathrm{p} \leq 0,0001)$ y rapidez de acción del fármaco $(\mathrm{r}=0,4131 \mathrm{p} \leq 0,0001)$.

Conclusión: Los aspectos relevantes en la satisfacción con el tratamiento fueron también los que más influian en la autoestima y la vida afectiva del paciente.

Palabras clave: Disfunción eréctil. Tratamiento médico. Calidad de vida.
\end{abstract}

\begin{abstract}
DETERMINING FACTORS IN PATIENT SATISFACTION WITH ERECTILE DYSFUNCTION TREATMENT

Introduction: Erectile dysfunction (ED) is associated with depression, anxiety and loss of self-esteem, deteriorating patient's quality of life. The present study aimed, to identify and evaluate key factors influencing patient's satisfaction with ED treatment. To analyze which of them have a greater impact on patient's self-esteem and satisfaction with different aspects of life.

Methods: The approach used was a multicentre, cross-sectional, epidemiological, observational study. The study group consisted of Spanish men over 18 years of age who received oral drug treatment for ED for at least 4 weeks before the study began. Pearson's correlation coefficient was calculated from the important aspects for the patient to assess his satisfaction with treatment ("Erectile Dysfunction Inventory of Treatment Satisfaction" (EDITS) questions 4-11) and overall satisfaction with treatment (EDITS question 1), the self-esteem subscale of the "Self-esteem and Relationship" (SEAR) questionnaire, and the "Fugl-MeyerLife Satisfaction Check List" (LISAT-8) questionnaire.

Results: A total of 1,646 patients were included. The most significant factors in connection with satisfaction with treatment for ED were; duration of the effects $(r=0.7311 \mathrm{p} \leq 0.0001)$, speed of action of the drug ( $\mathrm{r}=0.7088 \mathrm{p} \leq 0.0001)$, confidence in the ability to have sexual relations $(r=0.7021 \mathrm{p} \leq 0.0001)$ and partner satisfaction $(\mathrm{r}=0.692 \mathrm{p} \leq 0.0001)$. The factors which had greatest impact on self-esteem were duration of the effects $(r=0.6254 \mathrm{p} \leq 0.0001)$ and confidence in the ability to have sexual relations $(\mathrm{r}=0.6372 \mathrm{p} \leq 0.0001)$. The factors with the greatest impact on improvement in quality of life (Lisat-8 questionnaire) were: ease of use ( $\mathrm{r}=0.4372 \mathrm{p} \leq 0.0001)$ and $\mathrm{speed}$ of action of the drug $(\mathrm{r}=0.4131 \mathrm{p} \leq 0.0001)$.

Conclusion: The factors which most influenced satisfaction with the treatment were also those which had most impact on the patient's self-esteem and emotional life.
\end{abstract}

Keywords: Erectile dysfunction. Medical treatment. Quality of life.

Fuente de Financiación: Pfizer S.A. 
$\mathrm{L}_{\mathrm{a}}^{\mathrm{a}}$ a disfunción eréctil se define como la incapacidad de obtener y/o mantener una erección suficiente para lograr una actividad sexual satisfactoria $^{1}$. En España aproximadamente dos millones de varones con edades comprendidas entre los 25 y los 70 años presentan $\mathrm{DE}^{2}$.

La Organización Mundial de la Salud reconoce que la DE debe ser considerada como una enfermedad cuyos efectos van más allá de la vida sexual de los pacientes. La DE se encuentra asociada a estados de depresión, ansiedad y pérdida de autoestima $^{3,4}$ y afecta al bienestar y a la calidad de vida de las personas que lo padecen. Estos aspectos influyen negativamente en las relaciones sexuales con la pareja y en las relaciones con los familiares y amigos $^{1}$.

En los últimos años se han producido diferentes avances en el campo de las alternativas terapéuticas para la DE, sin embargo, son varios los autores ${ }^{5-8}$ que han corroborado que aún con estos avances, es todavía común que muchos de estos pacientes abandonen el tratamiento.

La repercusión del tratamiento de la DE no debería limitarse sólo a la obtención de una respuesta eréctil, sino que debería trasladarse a otros ámbitos de la vida del paciente.

Es importante, por tanto, valorar la efectividad de los tratamientos disponibles desde el punto de vista del paciente, mediante el uso de medidas de resultados sanitarios como la calidad de vida, el bienestar psicológico y el grado de satisfacción de los pacientes con su relación de pareja y con la vida en general $^{9-13}$.

Algunos autores han apuntado la relación positiva que existe entre la satisfacción con el tratamiento y la mejora de la autoestima y la calidad de vida $^{14}$. El presente estudio tuvo por objetivo, identificar y evaluar los aspectos más relevantes para el paciente en relación con la satisfacción con el tratamiento para la DE. analizando cuales de éstos tienen mayor impacto sobre la autoestima y la satisfacción con diferentes aspectos de la vida.

Por tanto, nuestra pregunta de investigación busca responder si todos los elementos evaluados por el paciente dentro de lo que denominamos "satisfacción con el tratamiento" tienen igual impacto sobre la mejora de la autoestima y la calidad de vida, o por el contrario, son sólo determinados aspectos, los que realmente están mejorando la per- cepción y el sentimiento de bienestar del paciente respecto a su autoestima y a su satisfacción con diferentes aspectos de la vida.

\section{PACIENTES Y MÉTODOS}

Estudio epidemiológico, transversal, multicéntrico y observacional. El estudio se realizó sin modificar la práctica clínica habitual del médico. Los médicos participantes, urólogos procedentes de todo el territorio nacional, seleccionaron a aquellos pacientes en tratamiento para la DE, que acudían a la consulta de uroandrología por cualquier causa. Todos los pacientes a los que se les ofrecía participar en el estudio debían, previo a su inclusión en el mismo, firmar el consentimiento informado. Se incluyeron en el estudio a hombres españoles mayores de 18 años, con diagnóstico previo de DE (puntuación en la dimensión "función eréctil" del IIEF <26), sin ninguna enfermedad mental, capaces de leer, entender y cumplimentar los cuestionarios, en tratamiento con cualquier fármaco oral para la DE durante al menos 4 semanas antes del comienzo del estudio y no más de 6 meses antes del inicio del mismo. Se realizó una única visita, para cada paciente, en la que se recogió toda la información. La inclusión de pacientes se realizó entre Enero y Julio de 2005.

El estudió se llevó a cabo siguiendo las normas de la Buena Práctica Clínica y conforme con los requerimientos y la Declaración de Helsinki, revisión de Escocia (Edimburgo, Octubre 2000). Cumpliendo con la legislación vigente, el protocolo fue revisado y aprobado por la Agencia Española del Medicamento.

Se evaluó el grado de DE de los pacientes al ser incluidos en el estudio mediante el Índice Internacional de Función Eréctil (The International Index of erectile Function, IIEF) ${ }^{15}$, cuestionario autoadministrado sencillo (15 preguntas), fiable y multidimensional, que ha demostrado su validez lingüística y psicométrica para explorar la función eréctil, en diferentes idiomas, entre ellos el español ${ }^{16,17}$. Consta de cinco dimensiones: función eréctil, función orgásmica, deseo sexual, satisfacción del acto sexual y satisfacción global derivada de la actividad sexual. La dimensión de la función eréctil está compuesta de 6 preguntas (de 1 a 5 y 15) con una puntuación total que va de 0 a 30 . El punto de corte es 25 , y define diferentes grados de DE: sin disfunción (26 a 30), disfunción leve (17 a 25), disfunción moderada (11 a 16) y disfunción grave/completa (1 a 10). 
La satisfacción con el tratamiento para la DE se valoró empleando la versión para el paciente, del cuestionario EDITS (Erectile Dysfunction inventory of treatment satisfaction) ${ }^{18}$ (Tabla 1). Las 11 preguntas se puntúan siguiendo una escala del 0 (muy insatisfecho) a 4 (muy satisfecho). La puntuación del cuestionario EDITS se calcula multiplicando por 25 la media de la puntuación de todas las preguntas originado una escala que va de 0 (satisfacción con el tratamiento extremadamente bajo) a 100 (satisfacción con el tratamiento extremadamente alto).

Se empleó la versión validada al español ${ }^{19}$ del cuestionario de autoestima y relaciones personales (The Self-esteem and Relationship, SEAR) ${ }^{20}$ fue diseñado para evaluar de forma específica la autoestima y calidad de vida en pacientes con ED. Consta de 14 preguntas con 5 posibles respuestas (Tabla 2), distribui-

Tabla 1. Cuestionario EDITS (versión del paciente)

1. En general, ¿está usted satisfecho con su tratamiento?

2. Durante las 4 últimas semanas ¿hasta que punto ha satisfecho el tratamiento sus expectativas?

3. ¿Considera probable continuar empleando este tratamiento?

4. Durante las 4 últimas semanas ¿̇le ha sido fácil emplear este tratamiento?

5. Durante las 4 últimas semanas ¿se ha sentido satisfecho con lo rápido que actúa el tratamiento?

6. Durante las 4 últimas semanas ¿Se ha sentido satisfecho con la duración de los efectos del tratamiento

7. Este tratamiento, ¿le ha hecho sentir confianza en sus propias posibilidades para mantener relaciones sexuales?

8. En general, ¿se siente satisfecha su pareja con los efectos del tratamiento?

9. ¿Que opina su pareja de que usted continúe con este tratamiento?

10. Durante las 4 últimas semanas ¿la erección ha sido natural?

11. Durante las 4 últimas semanas, ¿ha encontrado diferencias en la firmeza de erección con respecto a cuando usted no tenía problemas de erección?

Las posibles respuestas son específicas para cada pregunta (rango de 0 a 4):

0 = muy insatisfecho/nada $/$ muy improbable $/$ muy difícil de usar, etc.

4 = muy satisfecho/completamente/muy probable/muy fácil de usar, etc.

La puntuación del cuestionario EDIT se calcula multiplicando po 25 la media de la puntuación de las 11 preguntas. La puntuación final tendrá un rango que oscilará de 0 (la satisfacción más baja) a 100 (la mayor satisfacción)

Althof SE et al. Urology. 1999;53:793-799
Tabla 2. Cuestionario SEAR

1. Dominio de relaciones sexuales

Durante las 4 últimas semanas:

1) Me he sentido tranquilo al pensar en empezar cada relación sexual con mi pareja.

2) Me he sentido seguro de que mi erección duraría lo suficiente durante la relación sexual.

3) Me he sentido satisfecho de cómo he funcionado sexualmente.

4) He sentido que podría tener relaciones sexuales cuando surgieran de forma espontánea.

5) Me he sentido predispuesto a empezar yo las relaciones sexuales.

6) Me he sentido seguro de funcionar sexualmente.

7) Me he sentido satisfecho con nuestra vida sexual.

8) Mi pareja se ha sentido descontenta con la calidad de nuestras relaciones sexuales.

\section{Dominio de Confianza}

2a. Subescala de autoestima

9) Me he sentido bien conmigo mismo.

10) Me he sentido un hombre completo.

11) Estuve propenso a sentir que era un fracasado.

12) Me he sentido seguro de mi mismo.

2b. Subescala de Relacion en general

13) Mi pareja se ha sentido satisfecha con nuestra relación en general.

14) Me he sentido satisfecho con nuestra relación en general.

Opciones de respuesta:

$1=$ Casi nunca/nunca

2 = Pocas veces (mucho menos de la mitad de las veces).

$3=$ Algunas veces (aproximadamente la mitad de las veces).

4 = La mayoría de las veces (mucho más de la mitad de las veces).

$5=$ Casi siempre $/$ siempre

Capelleri JC et al. Int J of Impot Res. 2004;16:30-38

dos en dos dimensiones: Relaciones sexuales (preguntas 1-8) y confianza (preguntas 9-14). La dimensión de confianza se divide en dos subdimensiones: autoestima (preguntas 9-12) y satisfacción con la relación en general (preguntas 13-14). Tras invertir la puntuación de las preguntas 8 y 11 que se puntúan al revés, todas las puntuaciones son transformadas a una escala de 0 a 100, donde las puntuaciones mayores indican mayor autoestima y relaciones más satisfactorias.

La satisfacción del paciente con distintos aspectos de la vida, se evaluó mediante la versión validada al español del cuestionario LISAT-8 (Fugl-Meyer's Life Satisfaction of Life $)^{21,22}$. Este cuestionario, autoadministrado, consta de 8 preguntas, que se puntúan según una escala tipo Likert: 1 (muy insatisfecho), 2 (insatisfecho), 3 (algo insatisfecho), 4 (algo 
satisfecho), 5 (satisfecho), 6 (muy satisfecho). Las preguntas miden la satisfacción del paciente con 8 aspectos o facetas de su vida: vida general, vida sexual, relación de pareja, vida familiar, relación con amigos, ocio, situación laboral y económica (Tabla 3). La puntuación global va de 8 a 48 . Todas las preguntas pueden ser agrupadas en 3 dimensiones: Satisfacción con la faceta social de la vida (preguntas 1,4,5,6), satisfacción con la faceta afectiva de la vida (preguntas 2, 3) y satisfacción con la faceta laboral-económica de la vida (preguntas 7,8).

Todos los análisis estadístico fueron realizados empleando el paquete estadístico SAS ${ }^{\circledR}$ (versión 8.2). Se aplicó la correlación de pearson para valorar cual/cuales de las preguntas del cuestionario EDITS predecían mejor la satisfacción del paciente con su tratamiento. . La satisfacción con su actual tratamiento para la DE fue medido con la pregunta 1 del cuestionario EDITS “¿en general, como está usted de satisfecho con su tratamiento?”. Las preguntas 4 a 11 del cuestionario EDITS fueron los aspectos de importancia para ser valorados por los pacientes. Se realizó un análisis de correlación de Pearson entre las preguntas 4 a 11 del cuestionario EDITS y los cuestionarios SEAR y LISAT-8. La estadística descriptiva se realizó incluyendo medidas de tendencia central y dispersión para las variables cuantitativas, así como frecuencias absolutas y relativas para las variables cualitativas, con un intervalo de confianza del 95\% a dos colas en ambos casos.

Tabla 3. Cuestionario LISAT-8

¿Cómo son de satisfactorios los siguientes aspectos de su vida? Indique el número que mejor defina su situación

$1=$ Muy insatisfactoria
$2=$ Insatisfactoria
$3=$ Un poco insatisfactoria

$1:$ Mi vida en general es

2 : Mi vida sexual es

$3:$ Mi relación de pareja es

4 : Mi vida familiar es

5 : La relación con mis amigos y conocidos es

6 : Mi vida de ocio es

7 : My situación laboral es

8 : Mi situación económica es
$4=$ Un poco satisfactoria

$5=$ Satisfactoria

$6=$ Muy satisfactoria

Fugl-Meyer et al. Int J Impotence Res (1997) 9,141-148.

\section{RESULTADOS}

Un total de 318 urólogos procedentes de todo el territorio nacional participaron en este estudio. Se incluyeron 1.646 pacientes de los cuales fueron analizados los datos de 1.383 .

En la Tabla 4 se muestra las características sociodemográficas y clínicas de la población del estudio. Los pacientes del estudio presentaron una media de 2,5 años en el diagnostico de la DE. La mayoría de los pacientes $(73,6 \%)$ tenían estudios primarios o secundarios y aproximadamente la mitad de la población analizada se declaró fumador y/o consumidor de una media de 1,2 g de alcohol al día. Las principales enfermedades concomitantes fueron hipertensión $(40,8 \%)$ y dislipemia $(37,6 \%)$, con una media de la evolución de la enfermedad de $6,1 \pm 4$ y 5,2 $\pm 3,6$ años respectivamente. El 6,7\% de los pacientes había sufrido una cirugía radical de próstata, de éstos, el 51,5\% perdieron completamente las bandeletas neurovasculares ligadas a los nervios erectores del pene. Los principales tratamientos para la DE seguidos por los pacientes en el momento de ser incluidos en el estudio fueron: Sildenafilo $(70,4 \%)$, Tadalafilo $(9,2 \%)$ y Vardenafilo $(7,1 \%)$.

En la Tabla 5 se muestra la distribución de los pacientes según el grado de DE (según "el dominio de función eréctil” del IIEF) y el tratamiento recibido, así como la puntuación media global del cuestionario IIEF y de cada uno de sus dominios. La mayoría de los pacientes del estudio no presentaron DE $(22,7 \%)$ o ésta era de grado leve $(42,1 \%)$ Esto se correlaciona con las puntuaciones globales y por dominios del IIEF con una media de 43,3 $( \pm 15,7)$ para el total de la muestra, y una puntuación por grupos de $49,3( \pm 15,3)$ para el grupo de Sildenafilo, 46,7 $( \pm 15,6)$ para el de Tadalafilo y de 45,7 $( \pm 15,8)$ para el de Vardenafilo.

En la Tabla 6 se muestra el resultado total y por tratamientos de los cuestionarios EDITS, SEAR y LISAT-8. Respecto al grado de satisfacción con el tratamiento empleado (cuestionario EDITS), la mayoría de los pacientes $(78,3 \%)$ se encontró satisfecho con el mismo. 
Tabla 4. Características sociodemográficas y clínicas

\begin{tabular}{ll}
\hline Edad (años) & $59(9,9)$ \\
Peso (Kg) & $79,3(10,6)$ \\
Talla (cm) & $171,2(7,0)$ \\
IMC (kg/m²) & $27,1(4,2)$ \\
Fumadores (\%) & 46,5 \\
Consumo de alcohol (\%) & \\
No-consumidor & 33,7 \\
Ex-consumidor & 14,1 \\
Consumidor actual & 51,2 \\
Nivel educacional (\%) & \\
Sin estudios & 6,2 \\
Estudios primarios & 36,3 \\
Estudios secundarios & 37,3 \\
Estudios universitarios & 20,2 \\
Enfermedades concomitantes (\%) & \\
Hipertensión & 10 \\
Dislipidemia & \\
Diabetes & \\
Depresión & \\
Cardiopatia isquémica & \\
Neoplasias e hipertrofia prostática & \\
\hline & \\
\hline
\end{tabular}

Índice de Masa Corporal (IMC)

Los resultados se dan como media (desviación estándar) y

frecuencia relativa $(\%)$
La satisfacción con el tratamiento según el cuestionario EDITS fue de $85,9 \%$ para el grupo de Sildenafilo, 65,9\% para el grupo de Tadalafilo y $60,2 \%$ para el grupo de Vardenafilo, con diferencias estadísticamente significativas entre los grupos $(\mathrm{p}<0,001)$.

La puntuación total y por dominios del cuestionario SEAR, del conjunto de la población del estudio, fue mayor de 50 (Tabla 6). Las puntuaciones totales medias fueron de 70,1 $(17,5)$ para Sildenafilo, 60,1 $( \pm 19,7)$ para Tadalafilo y $58,9( \pm 22,5)$ para Vardenafilo, con diferencias estadísticamente significativas entre los grupos $(\mathrm{p}<0,05)$.

Globalmente, el nivel de de satisfacción con la vida (cuestionario LISAT-8) de los pacientes fue normal-alto (Tabla 6). Analizado por dominios, el dominio de satisfacción con la vida social (preguntas 1, 4, 5 y 6) alcanza puntuaciones mayores que los dominios de satisfacción con la vida afectiva (preguntas 2 y 3) y de satisfacción con la situación financiera (preguntas 7 y 8).

En la Tabla 7 se muestran el resultado de la correlación entre la satisfacción con el tratamiento (pregunta 1 del cuestionario EDITS) y los aspectos de importancia a la hora de valorar dicha satisfacción por los pacientes (preguntas 4 a 11 del cuestionario EDITS). La duración de los efectos, la rapidez de acción del fármaco y la confianza en poder

Tabla 5. Grado de DE según el dominio "Función erectile" del IIEF. Puntuación total y por dominios del IIEF según tratamientos

\begin{tabular}{|c|c|c|c|c|}
\hline & $\begin{array}{c}\text { Total } \\
(\mathrm{n}=1.351)\end{array}$ & $\begin{array}{c}\text { Sildenafilo } \\
(\mathrm{n}=956)\end{array}$ & $\begin{array}{c}\text { Tadalafilo } \\
(n=126)\end{array}$ & $\begin{array}{c}\text { Vardenafilo } \\
(n=94)\end{array}$ \\
\hline Grado de DE (\%) & & & NS & * \\
\hline Sin DE & 22,7 & 27 & 21,4 & 16 \\
\hline Leve & 42,1 & 43,5 & 40,5 & 48,9 \\
\hline Moderado & 18,4 & 16,2 & 21,4 & 17 \\
\hline Severo & 16,8 & 13,3 & 16,7 & 18,1 \\
\hline Puntuación IIEF\# & $43,3(15,7)$ & $49,3(15,3)$ & $46,7(15,6) \mathrm{NS}$ & $45,7(15,8)^{*}$ \\
\hline \multicolumn{5}{|l|}{ Puntuación Dominios\# } \\
\hline Función eréctil ( 1 a 30 ) & $19,1(7,4)$ & $20,1(7,2)$ & $18,5(7,3)^{*}$ & $18,6(7,3)^{*}$ \\
\hline Función orgásmica (0 a 10) & $7,0(2,8)$ & $7,3(2,7)$ & $6,8(2,8) \mathrm{NS}$ & $6,5(3,0)^{*}$ \\
\hline Deseo sexual (2 a 10) & $6,0(1,7)$ & $6,0(1,7)$ & $6,0(1,6) \mathrm{NS}$ & $5,9(1,8) \mathrm{NS}$ \\
\hline Satisfacción relación sexual (2 a 10) & $8,9(3,2)$ & $9,3(3,1)$ & $9,0(3,3) \mathrm{NS}$ & $8,6(3,2)^{*}$ \\
\hline Satisfacción en general (2 a 10) & $6,3(2,1)$ & $6,6(2,1)$ & $6,3(2,0) \mathrm{NS}$ & $6,1(2,1)^{*}$ \\
\hline
\end{tabular}

\#Los resultados se dan como media (desviación estándar); *P Sildenafilo vs otro tratamiento<0,05; no significante (NS); Desviación Estándar (DS); “The International Index of erectile Function” (IIEF); disfunción eréctil DE. 
Tabla 6. Puntuación de los cuestionarios EDITS, SEAR y LISAT-8

\begin{tabular}{|c|c|c|c|c|}
\hline & Total & Sildenafilo & Tadalafilo & Vardenafilo \\
\hline \multicolumn{5}{|l|}{ EDITS (rango puntuación 0-100) } \\
\hline Pacientes satisfechos (\%) & & & $* * * *$ & $* * * *$ \\
\hline No & 21,7 & 14,1 & 34,1 & 39,8 \\
\hline $\mathrm{Si}$ & 78,3 & 85,9 & 65,9 & 60,2 \\
\hline Puntuación total\# & $66,2(20,0)$ & $70,1(17,5)$ & $60,1(19,7)$ & $58,9(22,5)$ \\
\hline \multicolumn{5}{|l|}{ Puntuación por pregunta\# } \\
\hline Pregunta 1 & $66,8(25,0)$ & $70,9(23,0)$ & $61,9(24,6)^{* * * *}$ & $58,7(26,6)^{* * * *}$ \\
\hline Pregunta 2 & $62,2(25,4)$ & $66,6(23,0)$ & $55,4(24,6)^{* * * *}$ & $54,1(27,3)^{* * * *}$ \\
\hline Pregunta 3 & $73,4(29,4)$ & $79,7(25,1)$ & $65,1(29,2)^{* * * *}$ & $62,2(33,7)^{* * * *}$ \\
\hline Pregunta 4 & $79,0(24,3)$ & $83,1(20,4)$ & $72,4(27,2)^{* * * *}$ & $74,5(26,19 * * * *$ \\
\hline Pregunta 5 & $66,2(24,7)$ & $70,2(22,1)$ & $59,5(23,2)^{* * * *}$ & $62,0(27,6) * * *$ \\
\hline Pregunta 6 & $64,9(25,2)$ & $68,7(22,4)$ & $61,5(26,8)^{* *}$ & $57,7(29,3)^{* * * *}$ \\
\hline Pregunta 7 & $69,1(21,7)$ & $73,1(19,0)$ & $62,7(20,9)^{* * * *}$ & $60,5(26,1)^{* * * *}$ \\
\hline Pregunta 8 & $66,0(23,2)$ & $69,7(21,3)$ & $60,9(20,9)^{* * * *}$ & $58,2(26,1)^{* * * *}$ \\
\hline Pregunta 9 & $66,7(22,1)$ & $69,9(20,4)$ & $60,7(23,3)^{* * * *}$ & $59,9(24,8)^{* * * *}$ \\
\hline Pregunta 10 & $64,4(22,8)$ & $67,4(21,2)$ & $59,7(21,9)^{* * * *}$ & $57,9(25,0)^{* * * *}$ \\
\hline Pregunta 11 & $49,3(28,7)$ & $51,7(28,4)$ & $40,9(25,4)^{* * * *}$ & $42,3(27,7)^{* *}$ \\
\hline \multicolumn{5}{|l|}{ SEAR (rango puntuación 0-100) } \\
\hline Puntuación total\# & $60,7(21,8)$ & $63,6(21,0)$ & $57,4(20,3)^{* *}$ & $55,8(23,3)^{* * *}$ \\
\hline Puntuación dominio\# & $59,0(23,4)$ & $65,8(20,5)$ & $55,1(22,1)^{* * *}$ & $53,2(25,3)^{* * *}$ \\
\hline 1. Relación Sexual & $63,1(21,3)$ & $65,1(20,9)$ & $60,5(19,9)^{* *}$ & $59,2(23,2)^{* *}$ \\
\hline 2. Confianza & $62,5(21,5)$ & $67,2(23,1)$ & $59,5(20,0)^{* *}$ & $59,8(23,2)^{*}$ \\
\hline 2a. Autoestima & $64,3(24,2)$ & $62,4(25,4)$ & $62,3(23,1)^{*}$ & $58,0(26,0)^{* * *}$ \\
\hline 2b. Relación en general & $58,7(26,8)$ & $63,6(21,0)$ & $53,3(27,2)^{* * *}$ & $51,8(28,1)^{* * * *}$ \\
\hline Grado de satisfacción con su función eréctil & $66,1(17,0)$ & $68,3(16,2)$ & $63,6(18,3)^{* *}$ & $62,1(17,7)^{* * *}$ \\
\hline \multicolumn{5}{|l|}{ LISAT-8 (rango puntuación 0-100) } \\
\hline Puntuación total\# & $70,6(17,6)$ & $64,3(21,4)$ & $67-7(19,7)^{* *}$ & $66,1(19,8) * * *$ \\
\hline Puntuación dominio\# & $60,9(22,8)$ & $63,5(20,7)$ & $57,0(23,3) * * *$ & $56,7(24,5)^{* * *}$ \\
\hline Satisfacción con vida social (preguntas $1,4,5,6$ ) & $62,4(21,2)$ & $68,3(16,2)$ & $62,0(21,5) \mathrm{NS}$ & $59,6(23,0) \mathrm{NS}$ \\
\hline \multicolumn{5}{|l|}{ Satisfacción con vida afectiva (preguntas 2,3 ) } \\
\hline Satisfacción con situación financiera (preguntas & & & & \\
\hline
\end{tabular}

\#Los resultados se dan como media (desviación estandar);

****P sildenafilo vs tratamiento $<0,0001 ;{ }^{* * *} \mathrm{P}$ sildenafilo vs tratamiento $<0,001 ;{ }^{* *} \mathrm{P}$ sildenafilo vs tratamiento $<0,01$

*P sildenafilo vs tratamiento $<0,05$; No sigificante (NS)

EDIT puntuación (satisfacción con el tratamiento: 0=extremadamente bajo; 100= extremadamente alto)

SEAR puntuación (autoestima, relaciones : $0=$ extremadamente malas; 100= extremadamente buenas)

LISAT-8 puntuación

Tabla 7. Correlación entre la puntuación en la pregunta 1 y la puntuación en las preguntas 4 a 11 del cuestionario EDITS

\begin{tabular}{lc}
\hline Preguntas EDIT & r \\
\hline Pregunta 6: Duración & 0,7311 \\
Pregunta 5: rapidez & 0,7088 \\
Pregunta 7: confianza 0,7021 & \\
Pregunta 8: Satisfacción de pareja & 0,6929 \\
Pregunta 10: Naturalidad & 0,6072 \\
Pregunta 9: Opinión de la pareja & 0,5747 \\
Pregunta 11: firmeza & 0,4798 \\
Pregunta 4: facilidad de uso & 0,4384 \\
\hline
\end{tabular}

$\mathrm{P}$ valor $<0,0001$

Correlación de Pearson (r) mantener relaciones sexuales fueron los aspectos mas valorados por los pacientes $(r \geq 0,70)$. La satisfacción de la pareja, la naturalidad y la opinión de la pareja mostraron una correlación moderada $(0,69 \geq r \geq 0,5)$. Los aspectos menos valorados fueron la firmeza de las erecciones y la facilidad de uso $(r<0,50)$. Todas las correlaciones fueron estadísticamente significativas $(\mathrm{p}<0,0001)$.

En la Tabla 8 se muestra la correlación entre la subescala de autoestima del cuestionario SEAR y los factores determinantes de la satisfacción del paciente con el tratamiento (preguntas 4 a 11 del cuestionario EDITS). La autoestima de los paciente 
Tabla 8. Correlación entre la puntuación en el dominio de autoestima del cuestionario SEAR y la puntuación en las preguntas 4 a 11 del cuestionario EDITS

\begin{tabular}{lc}
\hline Preguntas EDIT & r \\
\hline Pregunta 7: Confianza & 0,6372 \\
Pregunta 6: Duración & 0,6254 \\
Pregunta 5: Rapidez & 0,6209 \\
Pregunta 8: Satisfacción de pareja & 0,6134 \\
Pregunta 10: Naturalidad & 0,5746 \\
Pregunta 9: Opinión de la pareja & 0,4974 \\
Pregunta 4: Facilidad de uso & 0,4609 \\
Pregunta 11: Firmeza & 0,4013 \\
\hline
\end{tabular}

$\mathrm{P}$ valor $<0,0001$

Correlación de Pearson (r)

presentó una correlación positiva-moderada con la confianza en poder mantener relaciones sexuales, la duración adecuada de los efectos y la rapidez del tratamiento, la satisfacción de la pareja y la naturalidad $(0,69 \geq \mathrm{r} \geq 0,5 \mathrm{p}<0,0001)$.

Respecto al análisis de cuales son los aspectos de la satisfacción con un tratamiento, que tienen un mayor impacto sobre la mejora global de la satisfacción con la vida, se observó una baja correlación $(0,3 \geq r \geq 0,49 \mathrm{p}<0,0001)$ para todos aquellos aspectos valorados (Tabla 9). Sin embargo, si se observó una moderada correlación entre el dominio afectivo de la vida y aspectos como la duración adecuada de los efectos, la satisfacción de la pareja, la confianza en poder mantener relaciones sexuales y la rapidez de acción del fármaco $(0,69 \geq \mathrm{r} \geq 0,5 \mathrm{p}<0,0001)$.

\section{DISCUSIÓN}

La función sexual y eréctil son consideradas componentes importantes del conjunto de la salud de los varones ${ }^{15}$. El impacto psicosocial de la DE puede conducir a estados depresivos, pérdida de la autoestima y dificultades para relacionarse con su entorno y con su pareja ${ }^{23,24}$. Un exitoso tratamiento de la DE puede mejorar la salud mental y social de los pacientes, así como su bienestar y autoestima ${ }^{24}$.

La satisfacción con el tratamiento en pacientes con DE, es muy importante para evitar el abandono de la terapia $^{25,26}$. La asociación demostrable entre la satisfacción con el tratamiento y factores psicosociales como la autoestima, confianza y relaciones personales, podrían sugerir que estos factores son también relevantes para el seguimiento del tratamiento ${ }^{26}$.

Diferentes estudios han analizado la relación entre la mejora de la función sexual y la satisfacción con el tratamiento ${ }^{27,28}$ o con la repercusión en la autoestima y relaciones sociales del paciente $\mathrm{e}^{29,30}$. Sin embargo, se han realizado pocos estudios en los que se evalúe globalmente la repercusión del tratamiento, analizando no sólo la eficacia clínica y la satisfacción con el tratamiento sino también su influencia en el ámbito psicosocial del paciente en su satisfacción con la vida ${ }^{24,31,32}$.

El presente estudio intenta profundizar un poco más, al analizar cuales son los aspectos importantes para el paciente a la hora de valorar el tratamiento, y cuales de ellos son los que afectan en mayor medida a su autoestima y diversos aspectos de su vida. Los resultados del estudio, parecen indi-

Tabla 9. Correlación entre la puntuación del cuestionario LISAT-8 (global y por dominios) y las preguntas 4 a 11 del cuestionario EDITS

\begin{tabular}{lcccc}
\hline Preguntas EDITS & LISAT-8 & Vida social & Vida afectiva & Vida económica \\
\hline Pregunta 4: Facilidad de uso & 0,4372 & 0,3857 & 0,4542 & 0,2764 \\
Pregunta 5: Rapidez & 0,4131 & 0,318 & 0,5651 & 0,1922 \\
Pregunta 7: Confianza & 0,4104 & 0,313 & 0,5792 & 0,1764 \\
Pregunta 6: Duración & 0,4102 & 0,302 & 0,5949 & 0,1773 \\
Pregunta 8: Satisfacción de pareja & 0,4082 & 0,3049 & 0,5985 & 0,1623 \\
Pregunta 9: Opinión de la pareja & 0,3709 & 0,2793 & 0,4961 & 0,1948 \\
Pregunta 10: Naturalidad & 0,3542 & 0,2631 & 0,5097 & 0,1536 \\
Pregunta 11: Firmeza & 0,2011 & 0,1064 & 0,3682 & 0,0737 \\
\hline
\end{tabular}

P valor $<0,0001$

Correlación de Pearson (r) 
car que aquellos aspectos que resultaron tener mayor influencia en la satisfacción con el tratamiento para los pacientes (duración adecuada de la erección, rapidez en hacer efecto el tratamiento, confianza en la capacidad de poder mantener relaciones sexuales y satisfacción de la pareja por el tratamiento) son los mismos que tienen mayor relación con la autoestima del paciente y con una satisfacción general con la vida y especialmente con el área afectiva de la misma. El hecho de saber que aspectos son los más importantes para los pacientes a la hora de valorar su tratamiento, permitiría poder hacer futuras valoraciones más exactas y eficaces.

Varios autores ${ }^{24,31}$ apuntan a la necesidad del uso de diferentes cuestionarios, cada uno de los cuales intentaría medir diferentes aspectos del perfil del pacientes modificados por el tratamiento para la $\mathrm{DE}$. De este modo, se podría lograr una completa valoración de cómo la terapia afecta al estado del paciente. En este sentido los cuestionarios empleados en el presente estudio, miden aspectos tales como la satisfacción del paciente con su tratamiento, su autoestima y la satisfacción con diferentes aspectos de la vida. Todo ello conduce a una visión bastante completa de la situación del paciente con DE en tratamiento. Los resultados del estudio indicarían que la mayoría de estos pacientes se encuentran satisfechos con el tratamiento, tienen un nivel de autoestima normal y están satisfechos con sus relaciones sexuales y con su vida en general. En el análisis por tratamientos, Sildenafilo presentó valoraciones más positivas que Tadalafilo y Vardenafilo. Estos resultados difieren de los observados por otros autores ${ }^{27}$ quizás debido a la propia naturaleza del estudio y el momento en el que fue llevado a cabo. Se podría decir que nuestros resultados se aproximarían más a la realidad clínica al corresponder a un estudio observacional, no intervencionista, realizado en una amplia muestra de pacientes. Quizás sería interesante poder comparar los resultados de nuestro estudio con los observados en otra población sana, de similares características, para poder corroborar que la DE tratada permite lograr una vida completamente normal.

\section{CONCLUSIONES}

Este estudio arroja que los aspectos relevantes en la satisfacción con el tratamiento fueron también los que más influían en la autoestima y la vida afectiva del paciente.

\section{REFERENCIAS}

1. National Institute of Health Consensus Development Panel on Impotence. Jama. 1993;270(1): 3-90.

2. Martín-Morales A, Sanchez-Cruz JJ, Saenz de Tejada I, Rodríguez-Vela L, Jiménez-Cruz JF, Burgos-Rodríguez R. Prevalence and independent risk factors for erectile dysfunction in Spain: results of the Epidemiología de la Disfunción Eréctil Masculina Study (EDEM). J Urol. 2001;166(2):569-574.

3. Feldman HA, Goldstein L, Hatzichristou DG, Krane RJ, McKinlay JB. Impotence and its medical and psychosocial correlates: results from the Massachusetts Male Ageing Study. J Urol. 1994;151(1):54-61.

4. Althof SE. Quality of life and erectile dysfunction. Urology. 2002;59(6):803-810.

5. Althof SE. When an erection alone is not enough: biopsychosocial obstacles to lovemaking. Int J Impot Res 2002;Suppl. 1:S99-104.

6. Montorsi F, Althof SE. Partner responses to sildenafil citrate (Viagra) treatment of erectile dysfunction. Urology. 2004;63(4): 762-767.

7. Mccullough AR, Barada JH, Fawzy A, Guay AT, Hatzichristou D. Achieving treatment optimization with sildenafil citrate (Viagra) in patients with erectile dysfunction. Urology. 2002; 60(2 Suppl 2):28-38.

8. Riley A. The role of the partner in erectile dysfunction and its treatment. Int J Impot Res. 2002;14:S105-109.

9. Althof SE, Turner LA, Levine SB, Risen C, Kursh ED, Bodner $\mathrm{D}$, et al. Intracavernosal injection in the treatment of impotence: a prospective study of sexual, psychological and marital functioning. J Sex Marital Ther. 1987;13(3):155-167.

10. Fugl-Meyer AR, Lodnert G, Bräholm IB, Fugl-Meyer KS. On life satisfaction in male erectile dysfunction. Int $\mathrm{J}$ Impot Res. 1997;9(3):141-148.

11. Hanson-Divers C, Jackson SE, Lue TF, Crwford SY, Rosen RC. Health outcome variables important to patients in the treatment of erectile dysfunction. J Urol. 1998;159(5):1541-1547.

12. Rosen RC. Sexual function assessment in the male: physiological and selfreport measures. Int J Impot Res. 1998;10(Suppl 2):S59-63.

13. Paige NM, Hays RD, Litwin MS, Rajfer J, Shapiro MF. Improvement in emotional well-beingand relationship of users of sildenafil citrate. J Urol. 2001;166(5):1774-1778.

14. Sánchez-Cruz JJ, Cabrera-León A, Martín-Morales A, Fernández A, Burgos R, Rejas J. Male erectile dysfunction and health-related Quality of Life. Eur Urol. 2003;44(2):245-253.

15. Rosen RC, Riley A, Wagner G, Osterloh IH, Kirkpatrick J, Mishra A. The International Index of Erectile Function (IIEF): a multidimensional scale for assessment of erectile dysfunction. Urology. 1997;49(6):822-830.

16. Rosen RC, Cappelleri JC, Gendrano N. The International Index of Erectile Function (IIEF): a state of the science review. Int $\mathrm{J}$ Impot Res. 2002;14(4):226-244.

17. Morales A, Mishra A, Conway K. Cross-cultural adaptation of the International Index of Erectile Function (IIEF). Latin American Branch of the International Society for Impotence Research. Annual Meeting, 1997, Linares, Chile.

18. Althof SE, Corty EW, Levine SB, Levine F, Burnett AL, McVary $\mathrm{K}$, et al. EDITS: Development of questionnaires for evaluating satisfaction with treatments for erectile dysfunction. Urology. 1999;53(4):793-799.

19. Rejas-Gutiérrez J, Cabello-Santamaría F, Calle-Pascual A, Chantada-Abad V, Fora-Eroles F, García-García M, et al. Validación de la versión española del cuestionario SEAR (SelfEsteem and Relationship Questionnaire) para varones con disfunción eréctil. Med Clin Barc. 2005;125(15):565-573. 
20. Capelleri JC, Althof SE, Siegel RL, Shpilsky, Bell SS, Duttagupta S. Development and validation of the Self-esteem and Relationship (SEAR) questionnaire in erectile dysfunction. Int $\mathrm{J}$ Impot Res. 2004;16(1):30-38.

21. Fugl-Meyer AR, Lodnert G, Bräholm IB, Fugl-Meyer KS. On life satisfaction in male erectile dysfunction. Int $\mathrm{J}$ Impot Res. 1997;9(3):141-148.

22. Rejas J, Gil de Miguel A, San Isidro C, Palacios G, Carrasco P. Adaptación cultural y validación psicométrica del cuestionario de satisfacción con la vida de Fugl-Meyer -LISAT 8- en población española masculina con disfunción eréctil. Pharmacoeconomics Sp Res Art. 2004;1:87-99.

23. Althof SE. Quality of life and erectile dysfunction. Urology. 2002;59(6):803-810

24. Cappelleri JC, Althof SE, Siegel RL, Stecher VJ, Tseng LJ, Duttagupta S. Association between the erectile dysfunction inventory of treatment satisfaction and the self-esteem and relationship questionnaire following treatment with sildenafil citrate for men with erectile dysfunction. Value in health 2005; (8Suppl 1):S54-60.

25. Mccullough AR, Barada JH, Fawzy A, Guay AT, Hatzichristou D. Achieving treatment optimization with sildenafil citrate (Viagra) in patients with erectile dysfunction. Urology. 2002;60 (2 Suppl 2):28-38.

26. Montorsi F, Althof SE, Sweeney M, Menchini-Fabris F, Sasso F, Giuliano F. Treatment satisfaction in patients with erectile dysfunction switching from prostaglandin E1 intracavernosal injection therapy to oral sildenafil citrate. Int $\mathrm{J}$ Impot Res. 2003;15(6):444-449.

27. Tolrá JR, Cuadrado JM, Fumadó L, Franco E. Prospective, randomized, open label, fixed-dose, crossover study to establish preference of patients with ED after taking the three PDE-5 inhibitors. J Sex Med. 2006;3(5):901-909.
28. Lewis R, Bennett CJ, Borkon WD, Boykin WH, Althof SE, Stecher VJ, et al. Patient and partner satisfaction with Viagra (sildenafil citrate) treatment as determined by the erectile dysfunction inventory of treatment satisfaction questionnaire. Urology. 2001;57(5):960-965.

29. Steidle C, Stecher V, Pace C, Tseng LJ, The SEAR Study Group. Correlation of improved erectile function and rate of successful intercourse with improved emotional well-being assessed with self-esteem and relationship questionnaire in men treated with sildenafil for erectile dysfunction stratified by age. Curr Med Res Opin. 2006;22(5):939-948.

30. Althof SE, O`Leary M, Cappelleri J, Glina S, King R, Tseng LJ Bowler JL. US and international SEAR study group. Selfesteem, confidence and relationships in me treated with sildenafil citrate for erectile dysfunction. J Gen Intern Med. 2006; 21:1-5.

31. Gil A, Martínez E, Oyagüez I, Palacios G, Rejas J. Erectile Dysfunction in Primary Care Setting: results of an observational, no-control-group, prospective study with Sildenafil under routine conditions of use. Int J Impot Res. 2001;13(6):338-334.

32. Martínez-Sánchez E, Oyagüez I, Carrasco P, Gil de Miguel A. Treatment with sildenafil and satisfaction with life in depressive patients with erectile dysfunction. Actas Esp. Psiquiatr. 2001; 29(5):293-298.

Correspondencia autor: Dr. J.A. Delgado Martín

Servicio de Urología. Hospital Clínico Universitario San Carlos Profesor Martín Lagos, s/n - 28040 Madrid

Tel.: 913303000

E-mail autor: jadelgadomartin@telefonica.net

Información artículo: Original - Disfunción eréctil

Trabajo recibido: mayo 2008

Trabajo aceptado: julio 2008 